\title{
A Bayesian Network Framework for Project Cost, Benefit and Risk Analysis with an Agricultural Development Case Study
}

\author{
Barbaros Yet ${ }^{\mathrm{a}^{*}}$, Anthony Constantinou ${ }^{\mathrm{b}}$, Norman Fenton $^{\mathrm{b}}$, Martin Neil $^{\mathrm{b}}$, Eike Luedeling ${ }^{\mathrm{c}}$, \\ Keith Shepherd ${ }^{\mathrm{c}}$
}

${ }^{a}$ Department of Industrial Engineering, Hacettepe University, Ankara, Turkey

${ }^{\mathrm{b}}$ School of Electronic Engineering and Computer Science, Queen Mary University of London, UK

${ }^{\mathrm{c}}$ World Agroforestry Centre (ICRAF), Nairobi, Kenya

Email Addresses: barbaros.yet@hacettepe.edu.tr (B. Yet), a.constantinou@qmul.ac.uk (A. Constantinou), n.fenton@qmul.ac.uk (N. Fenton), m.neil@qmul.ac.uk (M. Neil), e.luedeling@cgiar.org (E. Luedeling), k.shepherd@cgiar.org (K. Shepherd)

*Corresponding Author: Barbaros Yet

Department of Industrial Engineering, Hacettepe University, Beytepe Campus

Beytepe Ankara / Turkey 06800

E-mail: barbaros.yet@ hacettepe.edu.tr

Tel: $+903122978950 / 128$

\section{Abstract}

Successful implementation of major projects requires careful management of uncertainty and risk.

Yet such uncertainty is rarely effectively calculated when analysing project costs and benefits. This paper presents a Bayesian network (BN) modelling framework to calculate the costs, benefits, and return on investment of a project over a specified time period, allowing for changing circumstances and trade-offs. The framework uses hybrid and dynamic BNs containing both discrete and continuous variables over multiple time stages. The $\mathrm{BN}$ framework calculates costs and benefits based on multiple causal factors including the effects of individual risk factors, budget deficits, and time value discounting, taking account of the parameter uncertainty of all continuous variables. The framework can serve as the basis for various project management assessments and is illustrated using a case study of an agricultural development project.

Keywords: Project management, risk analysis, cost-benefit analysis, Bayesian networks 


\section{Introduction}

Projects provide a key mechanism that allows organizations to translate strategy into results. There is growing interest across many sectors in ways of becoming more time and cost effective or to demonstrate 'value for money' from projects. The need for improved organizational project management appears warranted. For example, the US Government Accountability Office indicated that approximately $72 \%$ of federal technology projects, amounting to a total budget of $\$ 27$ billion, are deemed to be poorly planned with the likelihood of encountering significant schedule and cost overruns (Mishra et al., 2016). The World Bank (2011) found from a review of 86 evaluations of its projects that as many as $41 \%$ had nonpositive outcomes. The World Bank expressed alarm over the fact that the percentage of their projects that are justified by cost-benefit analysis (which is perhaps the most basic of project assessment tools) has been declining for several decades and attributed this to a decline in adherence to standards and to difficulty in applying cost-benefit analysis (WorldBank, 2010).

Project management as a discipline has evolved from a focus on a single project to enhancing project management capacity within the whole organization. The concept of project management maturity has emerged as a measure of the level of capability or effectiveness of an organization in project management (Kerzner, 2001) and there are many project maturity models available (Backlund et al., 2014). A project maturity model usually defines different progressive levels of maturity, for example ranging from the lowest level of ill-defined project management processes applied by individuals on an individual project basis, to the most advanced level, which applies standard project management processes across an organization, uses quantified metrics to evaluate effectiveness and seeks out continuous improvement and innovation (Mishra et al., 2016, Spalek, 2014).

Uncertainty and risks are common elements of all major projects and they must be effectively managed for projects to be successful (Chapman and Ward, 2004, Ward and Chapman, 2003, Ward and Chapman, 1995, Green, 2001). Failure to account for uncertainty is a major cause of time and cost over-runs and disappointing project outcomes (Savage, 2012) but has been given insufficient attention in project 
management maturity. Project management maturity initiatives tend to seek improvements in management of risks, resources and time in isolation of one another, so that trade-offs are not apparent. Furthermore, treatment of risk in projects is commonly limited to using risk registers, which suffer a number of limitations, not least that they treat risks separately, as opposed to adopting a holistic approach that embraces a causal view of interconnected events (Fenton and Neil, 2012).

This paper focuses on uncertainty and risks associated with the cost, benefit and Return On Investment (ROI) of a project. We propose a Bayesian Network (BN) modelling framework that calculates these elements over the duration of the project, taking into account the uncertainty of all parameters while making these calculations. Our framework aims to model multiple risk events, and to enable users to assess the costs and benefits of the project under different risk scenarios. The model incorporates many important causal factors including the effects of having a budget deficit, uncertainty in cost estimates, time value of money, and the impact of inaccurate risk prediction. We illustrate the use of this framework using a case study of agricultural development projects.

Our approach complements previous work on project risk, which has focused on the planning and uncertainty of project time schedules, by focusing instead on costs and benefits of projects and the associated risk factors. Our model offers unique features by using uncertainty and variability of risk factors together with economic and adoption factors for making predictions in different time stages of a project. These features can help project managers especially in project selection, planning and control stages.

The paper is structured as follows: Section 2 provides an overview of BNs, which are proving to be an increasingly popular and effective method for modelling uncertainty and risk and reviews their previous applications in project management. Section 3 presents the proposed framework. Section 4 describes the case study and presents an instantiation of the framework for the case study. Section 5 shows the use and results of the model generated from the framework, and we provide our conclusions in Section 6. 


\section{Bayesian Networks}

Bayesian Networks $(\mathrm{BN})$ are powerful tools for making probabilistic inference on complex domains with a large number of variables (Fenton and Neil, 2012, Pearl, 1988). A BN is a probabilistic graphical model that consists of a graphical structure and parameters of conditional probability distributions corresponding to the structure. The graphical structure of a $\mathrm{BN}$ is composed of nodes representing variables, and arcs representing the relations between the variables. The parameters of a $\mathrm{BN}$ represent the nature and strength of the relations represented by the arcs. It is beyond the scope of this paper to show the technical details of BNs and their calculations; the readers are referred to Fenton and Neil (2012), and Koller and Friedman (2009).

The graphical structure of a BN is suitable for modelling causal relations (Pearl, 2000). Therefore, BNs offer unique features in integrating expert knowledge (Yet et al., 2014a, Yet et al., 2014b, Fenton and Neil, 2012) and data (Cheng et al., 1998, Heckerman, 1997) into model building. This is especially beneficial in domains where the availability of relevant data is limited but where extensive expert knowledge is available. As a result, BNs have been used for complex problems in many diverse domains including medicine (Yet et al., 2013, Yet et al., 2014b), law (Fenton et al., 2013, Fenton et al., 2014), finance (Neil et al., 2009), and sports (Constantinou et al., 2012, Constantinou et al., 2013).

Until recently, one of the main limitations of BNs was in building and calculating models that contain both discrete and continuous variables (such models are called hybrid BNs). Standard inference algorithms (such as the junction tree algorithm) and associated software packages require all variables to be either discrete or Gaussian. This turns out to be a major limitation for project risk models as they inherently contain many continuous variables that do not necessarily have a Gaussian distribution. However, recent advances in BNs, for example, the development of the dynamic discretization algorithm (Neil et al., 2007), have made it possible to build and solve hybrid BN models - involving arbitrary continuous distributions- accurately, efficiently and conveniently. These powerful algorithms have been 
implemented in a freely available BN software package with a graphical interface (Fenton and Neil, 2014, AgenaRisk, 2015). This software is used for the BN models in the paper.

\subsection{Bayesian Networks for Project Risk}

BNs are especially suited to model the attributes of uncertainty and risk that are common to all projects (as discussed in Section 1). BNs are powerful in reasoning about uncertainty as they are able to represent and make inference about complex joint probability distributions with numerous random variables. In addition to uncertainty and risk, all projects are unique by definition (PMI, 2013) and many, such as longterm development projects, have sparse relevant historical data. Moreover, the data collected from one project may not apply to others due to their differences and, it is often costly and time-consuming to collect data from long-term projects. However, expert knowledge is often available in abundance both in project management and the application domain and when available, it can be profitably used as a source of evidence (Shepherd et al., 2015). BNs offer powerful and unique features to use and combine this expert knowledge with available data, where available (Yet et al., 2014a, Renooij, 2001, Neil et al., 2000).

Despite their clear potential benefits, the use of BNs in project management has been quite limited (possibly because of the previous limitations on hybrid BNs). The earliest published article devoted to using BNs explicitly in a general project management context appears to be Khodakarami et al. (2007), which proposes a BN model to deal with the uncertainty in project scheduling. This model implements the critical path method (CPM) into a BN model, and extends CPM by reasoning with the causes of delays. Similarly, Luu et al. (2009) compute the risk of having an overall schedule delay using a discrete BN model without parameter uncertainty. Fineman et al. (2009) use a simple BN model to reason about the trade-off between the time, cost and quality aspects of a project. Lee et al. (2009) use a BN model to estimate the risk of exceeding budget and time schedule, and of having insufficient specifications. They apply their model in the shipbuilding domain. Khodakarami and Abdi (2014) use BNs to estimate only the project costs based on the causes of the costs. 
In contrast to the relatively few applications of BNs to general project management, there has been more extensive use in the specific context of management of software engineering projects (possibly because of the proximity of this domain to computer scientists). Fan and $\mathrm{Yu}$ (2004) proposed a framework that continuously assesses and manages risk in different aspects of software development. Fenton et al. (2004) demonstrate a static BN model for making resource decisions in software projects. Their model takes the trade-off between quality, time and costs into account, and it is able to make inference about the resources required to achieve a target quality value. de Melo and Sanchez (2008) use discrete BNs to assess risks and predict delays in software maintenance projects. Hu et al. (2013) use constraint-based structure learning algorithms on $\mathrm{BNs}$ to learn causal relations and make predictions about the risk factors of software development projects. Perkusich et al. (2015) use BNs to identify problematic processes in software development projects.

Among the reviewed studies, the most similar ones to our framework are (Lee et al., 2009) and (Fenton et al., 2004). Lee et al.'s model (2009) is suitable for making a cost, benefit and ROI analysis of a project. The model has a wider scope that also predicts schedule delays but its structure is a discrete and static $\mathrm{BN}$ that contains a small number of nodes. As a result, their model does not calculate the uncertainty of continuous parameters and changing risks in different time stages. Fenton et al.'s model (2004) reasons about the cost and quality trade-off, and calculates the effects of budget and individual risk factors on project outcomes. Their model contains continuous variables that take parameter uncertainty into account. However, Fenton et al. uses a simpler, static, model that has aggregate cost, quality and time values for the whole project. Both Lee et al. (2009) and Fenton et al. (2004) developed their models for a specific domain. In this paper, we propose a general framework that calculates the costs, returns, and the effects of risk factors in individual time stages of a project by taking both the uncertainty of parameters and the variability of risks into account. In the following section, we describe the structure of our modelling framework. 


\section{Overview of Framework}

Since one of our objectives is to calculate the return on investment over multiple years, our framework is based on a Dynamic Bayesian Network (DBN) that represents individual time stages $t$ with separate linked BN objects. In DBNs, each BN object is a BN model structure that represents the variables and relations between them at each predetermined time stage. The BN objects are linked together through the nodes that represent temporal relations over different time stages. The purpose of modelling with BN objects is to simplify inference over multiple time stages, and to clarify the structure and parameters of the model in different time stages (Murphy 2002). In our framework, the structures of the BN objects are identical for the first time stage and onwards, i.e. $t=1,2, \ldots, n$. Only the structure of the BN object corresponding to the beginning of the project $(t=0)$ is different from the others.

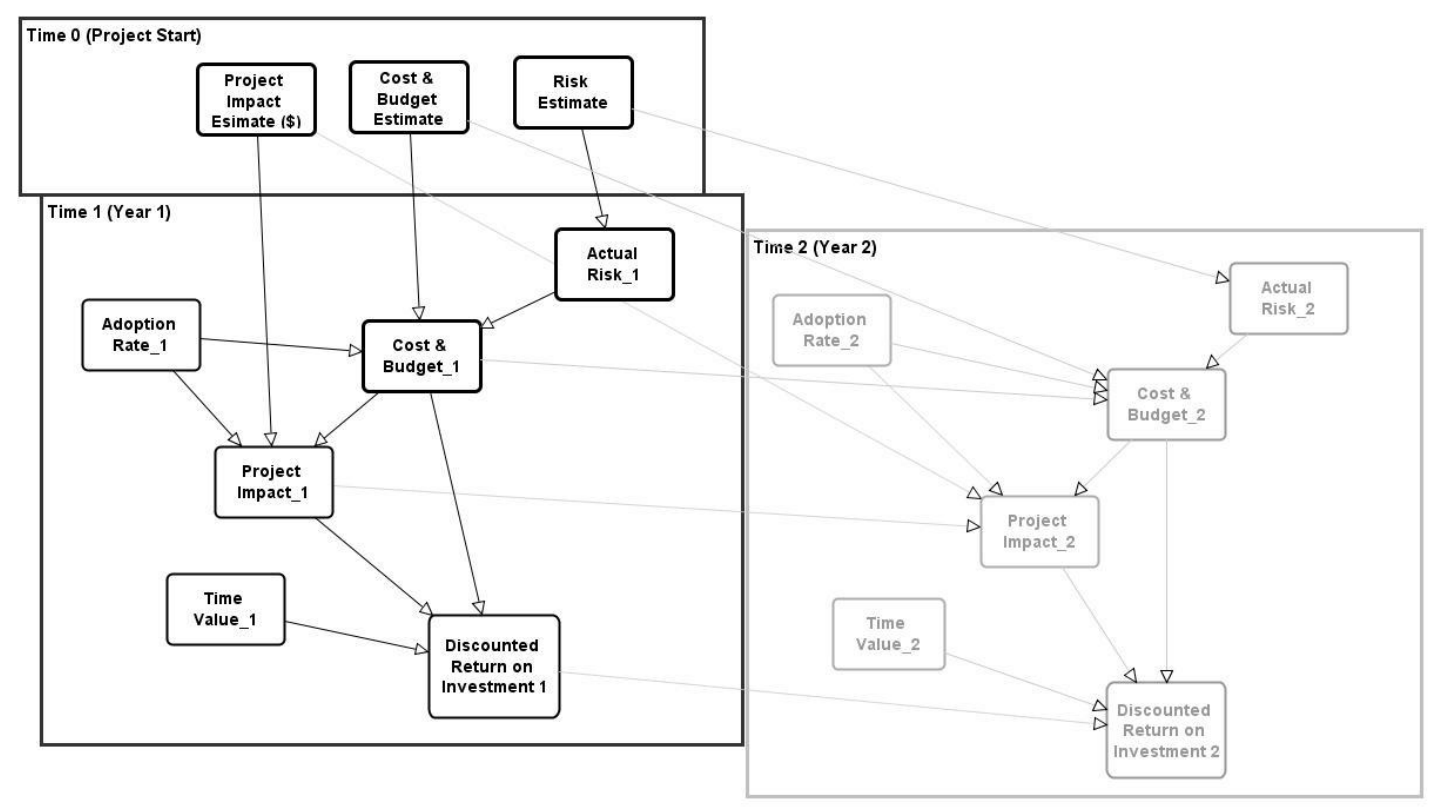

Figure 1 Template Model

The BN objects corresponding to individual time stages can have large structures in our framework. In order to simplify description and building of these objects, we also divided the BN structure of each BN object into smaller fragments of model structures. We call these smaller fragments 'BN components'. A $\mathrm{BN}$ component consists of multiple nodes and arcs that represent a similar purpose or concept in the BN 
object. For example, all nodes relevant to costs calculations in a time stage $t$ can be located in a $\mathrm{BN}$ component named 'Cost \& Budget $t$ '. BN components do not have any use when the model is calculated; their only purpose is to clarify and help describe and build the BN. In summary, our framework is divided into multiple $\mathrm{BN}$ objects, each of which represents a distinct time stage, and each $\mathrm{BN}$ object can be divided into multiple BN components each representing a similar purpose or concept in the time stage.

Figure 1 shows the template of our BN framework (for the first three years of a project; the extension to subsequent years follows the same pattern). The rectangle nodes shown in Figure 1 are BN components that contain multiple nodes and arcs. The content of these components may change in different domains. For example, the content of 'cost \& budget estimate' component in the Time 0 object can differ between a construction project and a software project. Therefore, the structure shown in Figure 1 is not a BN but its aim is to show the relations between different components and serve as a template to build such BN models. We use the term template to illustrate that the contents of each component can be defined as the user sees fit, to match local conventions, and still be coupled together using this process.

In the remainder of this section the $t=0$ (Section 2.1) and $t=1,2, \ldots, n$ (Section 2.2) BN objects and the $\mathrm{BN}$ components within these $\mathrm{BN}$ objects.

\subsection{Time 0 (Project start)}

The 'Project Start' BN object models the cost, budget, impact and risk estimates prepared at the beginning of the project (see the top of Figure 1). Each rectangle node within this BN object in Figure 1 represents a BN component that contains multiple nodes and variables. The content of these components is examined in the remainder of this section. 


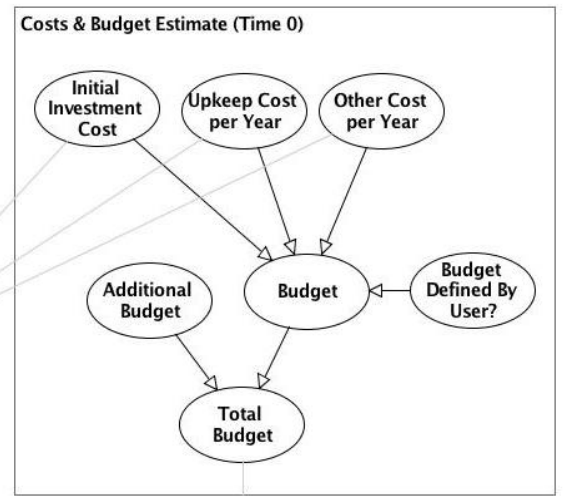

Figure 2 Cost and Budget Estimate

\subsubsection{Cost \& Budget Estimate}

The 'cost \& budget estimate' component contains nodes representing different kinds of costs and budget.

Figure 2 shows the BN structure corresponding to the 'Cost \& Budget Estimate' component in Figure 1. Specifically,

a) the 'initial investment cost' node represents one-off capital cost spent at the beginning of the project,

b) the 'upkeep cost per year' node represents the yearly costs such as personnel and materials. In later time stages, this estimate is adjusted with the percentage of people who adopt the outcome of the development project in order to calculate the actual upkeep cost at that stage (see Sections 2.2.2 and 2.2.3), and

c) the 'other cost per year' node includes the costs associated with risks. These costs are adjusted in later time stages depending on the discrepancy between the estimated and actual risks (see Section 2.2.1).

The three categories of costs above were chosen because they are common to most projects. However, additional categories can be added by modifying the structure shown in Figure 2. For example, we can model upkeep cost in more detail by adding separate nodes for labour and material cost as a parent of the 'upkeep cost' node. 


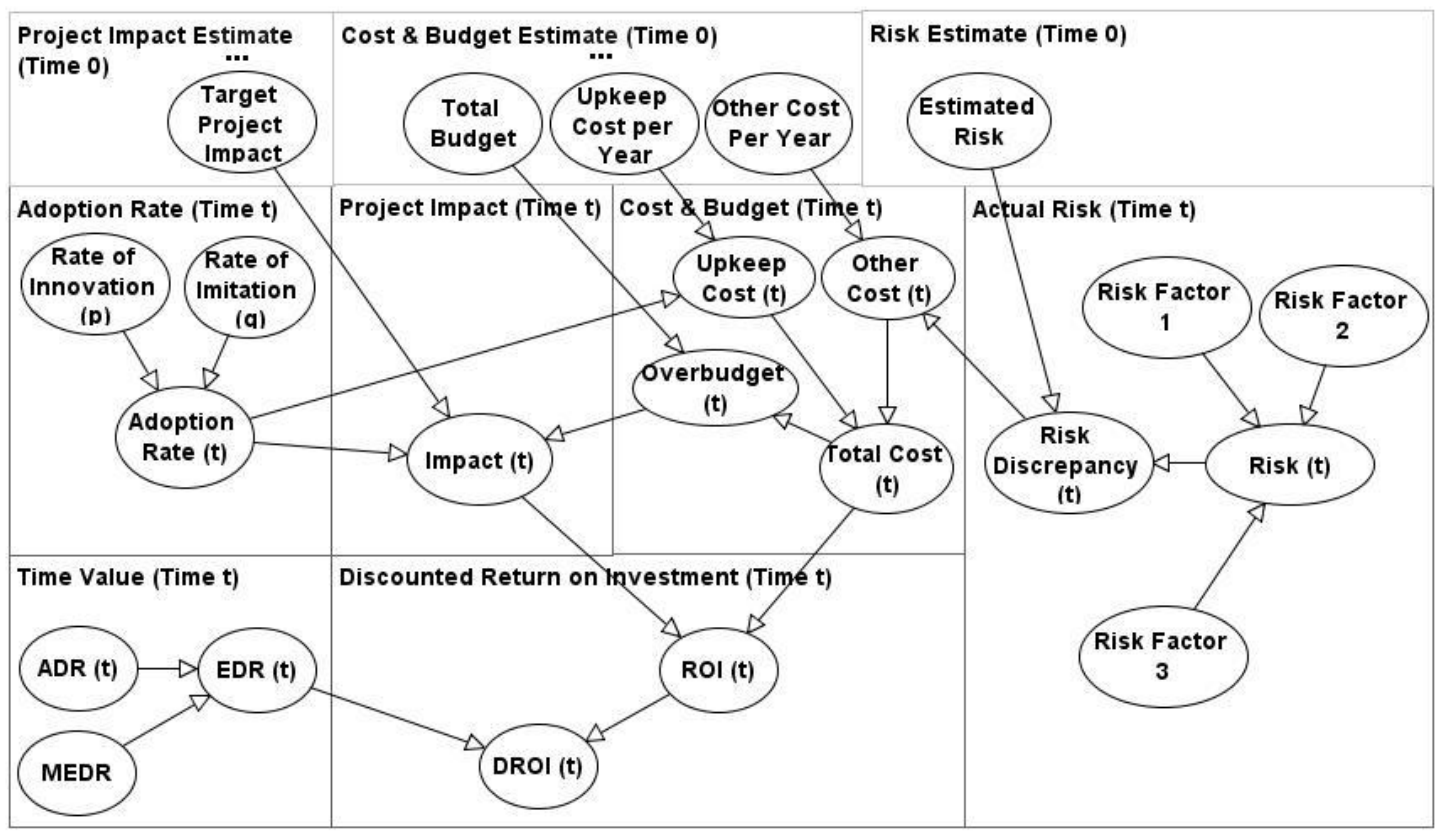

Figure 3 Components of the Framework

The 'budget' node estimates the initial budget by summing up the initial investment, upkeep and other costs by taking project duration into account. The project duration is defined in the parameters of this node. Alternatively, the user can manually define the budget by entering a point value or a distribution. The BN offers the flexibility for the budget to be estimated from costs or user-defined. The 'additional budget' node represents the additional capital that can be invested in the project; crucially, in a fixed cost project this will be set to zero. This node can have either a point value or a distribution. We can also use the $\mathrm{BN}$ model to automatically calculate the extra budget needed to avoid having a budget deficit. If extra cost $i s$ required but no extra budget is available in later time stages (so additional budget is still set to 0) then we will observe a decrease in the impact of the project as a result of the budget deficit.

The 'total budget' node sums up the initial budget and additional budget. This variable is used in later time stages to calculate the presence and impact of budget deficits.

\subsubsection{Project Impact Estimate}

The 'project impact estimate' component models the targets for different categories of impact expected from the project. Although impact is often transformed to monetary value, categories of impact are likely 
to differ in different domains. For example, the impact categories in a software project are different from the categories in an agricultural development project.

We adjust the impact for stakeholders as the utilities of the project impact can differ for different stakeholders. For example, the utilities of additional income for people with high and low income can be different. In that case, we can adjust the impact by an income utility multiplier that models the utility of marginal income as a decreasing function of the income. Consequently, the benefits of a project for different stakeholders can be calculated.

In later time stages, the cost estimate and target impact is adjusted by adoption rate (a concept that we discuss in Section 2.2.2) and the degree of budget deficit to calculate the cost and impact in a particular time stage. In other kinds of project added value measures could be used equally well.

\subsubsection{Risk Estimate}

The 'risk estimate' component models the initial risk estimated at the beginning of the project. In subsequent time stages, estimated risk is compared to the observed risk factors to adjust the costs (see Section 2.2). One of the main objectives of the model is to account for project risks as they are observed, so the question is: to what extent have any of the risks already been considered in the initial budget and target impact. For example, an initial risk estimate can be modelled by a variable that has five ordinal states ranging from 'Very Low' to 'Very High'. If no risks have been considered, then the 'risk estimate' will be assigned the value 'Very Low'. If there are actually no real project risks, then the 'actual risk' (the variable in the model that takes account of known risks at any stage) will also be 'Very Low' and there will be no 'discrepancy' between the estimate and actual risks. If, however, there are tangible project risks then there will be a positive discrepancy that will result in the model predicting increased required costs in order to meet the target impacts. 


\subsection{Time $t=1,2, \ldots, \mathrm{n}$}

In each time stage $t=1,2, \ldots, n$, our model calculates the cost, impact, and total return on investment. In the remainder of this section, we describe the structure of the components in a 'Time $t$ ' object (see Figure 1).

\subsubsection{Actual Risk at time $t$}

The framework compares the observed risks to the estimated risks in each time stage. Individual risk factors observed at time $t$ are entered. If the risks are over or under estimated, actual costs are respectively higher or lower than the estimated costs. We model this reasoning by adjusting the estimated costs by the degree of discrepancy between the estimated and actual risk (see Figure 3).

Ranked nodes (Fenton et al., 2007) offer a flexible and convenient way of modelling the degree of estimated and actual risks. A ranked node is an approximation of the truncated normal distribution to the multinomial distribution with ordinal scale. As a result, a ranked node automatically transforms the ordinal states of the risk estimates into numerical values based on the truncated normal distribution. Moreover, ranked nodes have many associated functions that can be used to model the behaviour between individual risk factors and an overall summary of risk. For example, a summary risk node can be modelled as a weighted average of individual risk factors when both summary and individual risk nodes are modelled using a ranked node. Fenton et al. (2007) provides a convenient approach to elicit model parameters for ranked nodes. The ordinal states of a ranked node must be carefully defined with domain experts in order to correctly represent the risks of the application domain. Continuous variables should be used when the behaviour of a risk node is not suitable to be modelled by using ordinal states. Our framework is able to integrate continuous factors together with ranked nodes.

\subsubsection{Adoption Rate at time $t$}

The project impact and direct costs can also be adjusted by adoption rate, which is an important component in most application domains of project management. The adoption rate represents the rate of expected end use of the outcome of the project (which could be a product, a new technology or an 
innovation) within a specified period of time. For example, in all commercial product design projects, the impact of the project increases as more people adopt the product developed by the project. This is certainly also true of the case study in Section 4 (agricultural development projects). The Bass model is commonly used to model adoption rate (Bass, 1969). It uses rate of innovation $p$ and rate of imitation $q$ to estimate the rate of adoption $A R$ over a specified time period $t$ as follows:

$$
A R=\frac{1-e^{-(p+q) t}}{1+\left(\frac{q}{p}\right) e^{-(p+q) t}}
$$

Our framework allows us to define $p$ and $q$ by using either a point value or an entire probability distribution (see Figure 4). If adoption rate is not relevant to the application domain, the framework can be conveniently adapted by either removing the adoption rate component or entering a fixed adoption rate of one in this component.
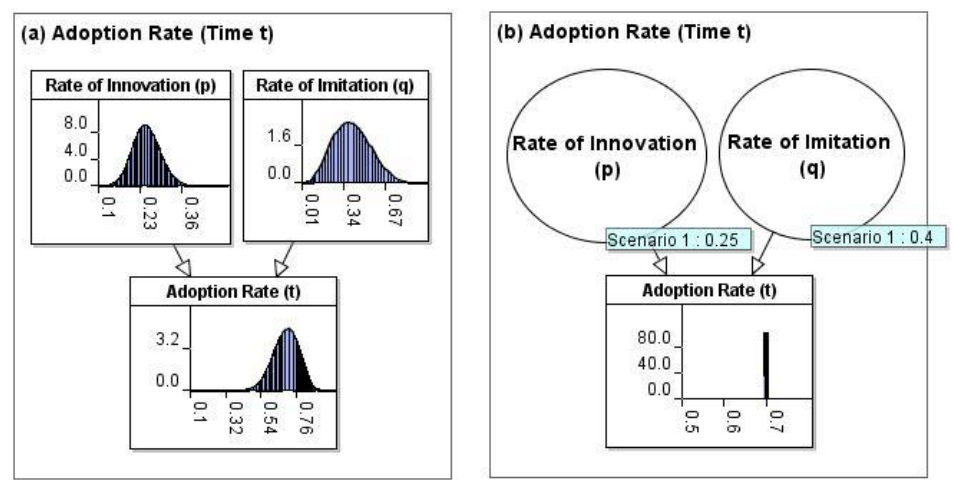

Figure 4 Adoption rate parameters defined as (a) probability distributions and (b) point values

\subsubsection{Costs and Budget at $t$}

This component calculates the total cost at time $t$ by summing different categories of costs, such as upkeep and other costs (see Figure 3). The upkeep and other costs are adjusted by the adoption rate (Section 2.2.2) and risk discrepancy (Section 2.2.1) respectively. The total cost is compared with the project budget at each time stage. If the total cost is greater than the budget at time $t$, the project impact at that stage is reduced according to the extent of exceeding the budget. 
At each time step, the BN model calculates the probability of running over-budget by comparing the costs to the total budget of the project. If the project costs are higher than the total budget of the project, this will have a negative effect on the impact of the project. The actual impact will decrease, and therefore be smaller than the estimated impact, as the budget deficit increases. The framework is flexible in terms of modelling the relation between budget deficit and impact: we can use a linear, exponential or similar expression to model the behaviour of this adjustment.

\subsubsection{Project Impact at time $t$}

The project impact is calculated by adjusting the estimated impact per year by the adoption rate (Section 2.2.2) and the degree of budget deficit (Section 2.2.3) at $t$ (see Figure 3).

\subsubsection{Discounted Return on Investment and Time Value at time $t$}

The framework calculates the ROI by subtracting the total cost from the impact at every time stage $t$ (see Figure 3). Since the value of money diminishes as the time progresses, we discount the ROI. We can use a wide variety of approaches to calculate Discounted ROI (DROI) including the fixed and environmental discount rate. A Fixed Discount Rate (FDR) is calculated as follows:

$$
F D R=\frac{1}{(1+A D R)^{t}}
$$

where ADR is the annual discount rate.

Environmental Discount Rate (EDR) can be better suited to long-term development and environmental projects as FDR can underestimate long-term benefits. EDR shows the behaviour of a fixed discount rate in the short term but the discount rate asymptotically approaches to a minimum value in the long term. EDR for year $t$ is calculated as follows:

$$
E D R=\frac{1}{(1+A D R)^{t}}(1-M E D R)+M E D R
$$

where MEDR is the minimum environmental discount rate. Both ADR and MEDR can be defined by either a point value or a probability distribution in our framework. 
In the following section, we present a case study to which we apply this framework. For a specific application, the variables in the project impact, cost and budget estimate and risk estimate modules will need to be disaggregated further, requiring additional nodes and NPTs, but the basic higher level structure of the framework is generalisable. The purposes of the case study are to illustrate the methodology rather than validate the expert estimates or predictions made during the project itself.

\section{Case study set-up and model}

Although the overall structure of the framework (Figure 1) is the same for most domains, instantiation of the components, and the associated templates, may differ. Our case study is based on an agricultural development project. This will obviously have different risk factors and pathways to impact than, for example, a software project. Therefore, the BN components about these factors must be built considering the specifics of the application domain. Section 4.1 presents the case study and Section 4.2 describes the parts of the framework that are modified for the case study.

\subsection{Agricultural development project}

In the case study we collaborated with relevant domain experts to build a model to calculate the costs, impact and ROI of a generic agricultural development project. The primary risk factors that can significantly affect the outcome of such a project are: extreme climate events, political instability of the country where the project is done, degree of stakeholder involvement, and efficacy of the enabling organisation. These risk factors are defined by a group of domain experts. The project can benefit the local community by improving the quality of food and water supplies. The impact to the local population will increase as more people adopt the development outcomes of the project. A project may also have environmental benefits by decreasing the ecological footprint. A one-off capital investment is made at the beginning of the project. The project will also have yearly upkeep costs for the employees and materials used. All other costs, including the costs due to risk events, will be categorised as 'other costs'. In the remainder of this section, we fine-tune the framework to model the specifics of the case study. In Section 5, we will parameterise the model and calculate ROI for a 10-year period. 


\subsection{Fine-tuning for Case Study}

The case study has impact categories that are unique to development projects. Therefore, we need to build the 'project impact estimate' component at time 0 specifically for the case study (Section 4.2.1). The initial risk estimate component also needs minor modifications for the case study (Section 4.2.2). Finally, we need to modify the 'actual risk' component, as risk factors are also specific to the domain (Section 4.2.3). We use the Bass model and EDR to model the adoption and discount rate respectively. We therefore do not need to modify the structure of these components for the case study. In the remainder of this section, we describe the components that are modified for the case study.

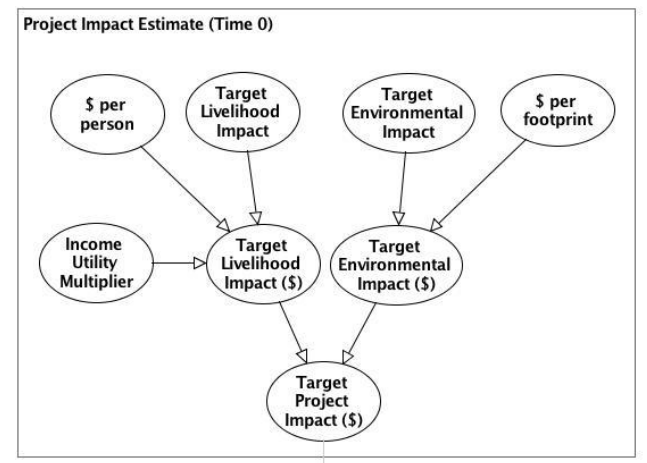

Figure 5 Project Impact Estimate in Case Study

\subsubsection{Project Impact Estimate}

Figure 5 shows the 'Project Impact Estimate' component refined for the case study. An agricultural development project is assumed to have two different kinds of impact: environmental and livelihood impact. These impacts are transformed into monetary values in US dollars per person and in US dollars per environmental footprint respectively.

For livelihood impact, we also consider the difference of the utilities of additional income for people with high and low income. We assume the utility of marginal income as a decreasing function of the income, and adjust the livelihood income accordingly. Using an income utility multiplier allows us to calculate the benefits of models for different stakeholders. The target project impact component estimates the overall target by summing up the livelihood and environmental targets. The target project impact is adjusted by the adoption rate and degree of budget deficit in later stages. 


\subsubsection{Risk Estimate}

Figure 6 shows the estimated and actual risk components. The 'estimated risk' variable is modelled with a ranked node that has five ordinal states ranging from 'Very Low' to 'Very High'. This variable represents the initial risk assessment done during the planning stage. We also added a variable representing the country where the project is done. This variable is used in later time stages to estimate the risk due to political instability of the country.

Table 1 Input Variables and Distributions for Project Proposals

\begin{tabular}{lll}
\hline \multicolumn{1}{c}{ Variable } & Proposal 1 & \multicolumn{1}{c}{ Input Values / Distributions } \\
& 15000 & $\operatorname{Proposal} 2$ \\
\hline Initial Budget & $\operatorname{TNormal}(3750,1000,0,10000)$ & $\operatorname{TNormal}(1000,250,0,10000)$ \\
Initial Investment Cost & $\operatorname{TNormal}(300,250,0,3000)$ & $\operatorname{TNormal}(280,100,0,3000)$ \\
Upkeep Cost / Year & $T N \operatorname{Nomal}(1000,500,0,10000)$ & $\operatorname{TNormal}(290,100,0,10000)$ \\
Other Cost / Year & 20 & 10 \\
Target livelihood impact & 100 & 35 \\
Target environmental footprint & 20 \\
KUSD / population year & 20 & 40 \\
KUSD / footprint year & 40 & Uganda \\
Country & Kenya & Medium \\
Risk estimation & Medium & $\operatorname{Beta}(30,70)$ \\
Rate of innovation $(\boldsymbol{p})$ & $\operatorname{Beta}(25,75)$ & $\operatorname{Beta}(50,50)$ \\
Rate of imitation $(\boldsymbol{q})$ & $\operatorname{Beta}(4,6)$ & $\operatorname{TNormal}(0.03,0.001,0,1)$ \\
ADR & $\operatorname{TNormal}(0.03,0.001,0,1)$ & $\operatorname{Beta}(26,74)$ \\
MEDR & $\operatorname{Beta}(26,74)$ & 1.57 \\
Income utility multiplier & 1.57 & \\
\hline
\end{tabular}

\subsubsection{Actual Risk}

Each main risk factors identified by the domain experts (political instability, extreme climate impact, institutional efficacy and stakeholder involvement) is modelled with a ranked node as a parent of the "risk (t)' node representing the actual risk at $t$. The 'risk (t)' node is also a ranked node defined by a weighted average of the individual risk factors. 


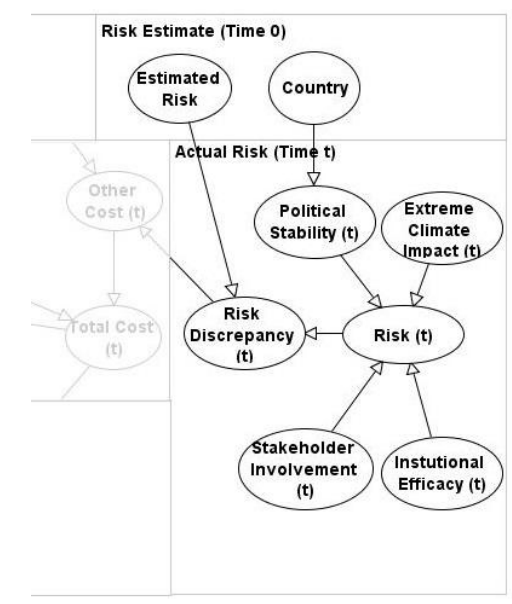

Figure 6 Estimated and Actual Risk in Case Study

\section{Case study results}

While Section 4 demonstrates the ease with which the generic framework could be tailored to a specific application domain, this section presents the use and results of the tailed version of the model described there. We calculate costs and benefits of two alternative agricultural development project proposals over a period of 10 years. One of these proposals has higher costs and uncertainty but it can also lead to higher impact. The other project is a safer option requiring a smaller budget but its potential return is also smaller. We analyse the potential return on investment and effects of adjusting the budget of these projects under different risk scenarios.

\subsection{Initial Analysis}

Our first step is to enter the initial budget, cost, impact and risk estimates to the model for each proposal. The unit of all monetary values is in thousand US dollars (KUSD). Some of our estimates are point values, others are probability distributions. For example, we use a point value for our budget estimate, i.e. initial budget of the first proposal is 15000 KUSD, and a truncated Normal distribution for our upkeep cost estimate, i.e. TNORMAL $\left(\mu: 300, \sigma^{2}: 250, a: 0, b: 3000\right)$ where $a$ and $b$ are lower and upper bounds respectively. Our modelling framework is flexible in enabling us to use point estimates or probability distributions to define any parameter in the model. The parameters of a BN model can be elicited from domain experts (Renooij, 2001), learned from data (Daly et al., 2011) or defined by using a combination 
of both (Yet et al., 2014a). Table 1 shows the values and distributions used for initial parameters of each proposal.

\subsection{Cost-Benefit Calculations}

After entering the initial parameters for each proposal, we run the model to calculate the costs, benefits and DROI for a 10-year period. The model provides detailed information about these factors including their entire probability distributions. For example, we are able to obtain the probability distributions of cost, impact and return on investment for each year. Moreover, the model estimates the risk and impact of running over-budget in different years (see Figure 7).

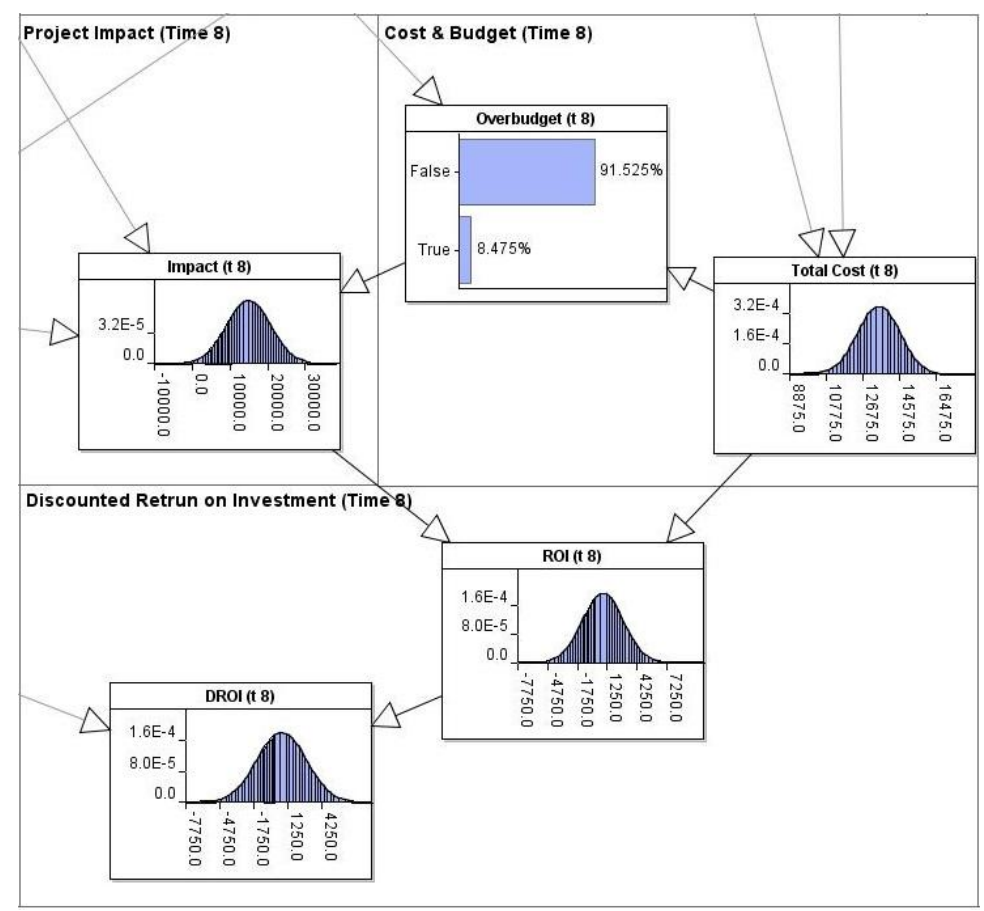

Figure 7 Probability distributions of the cost, impact and ROI estimates of the model

Figure 8 shows the risk of exceeding budget (REB), and the $95 \%$ credible interval (CI) of discounted return on investment (DROI) for proposals 1 and 2 respectively. 

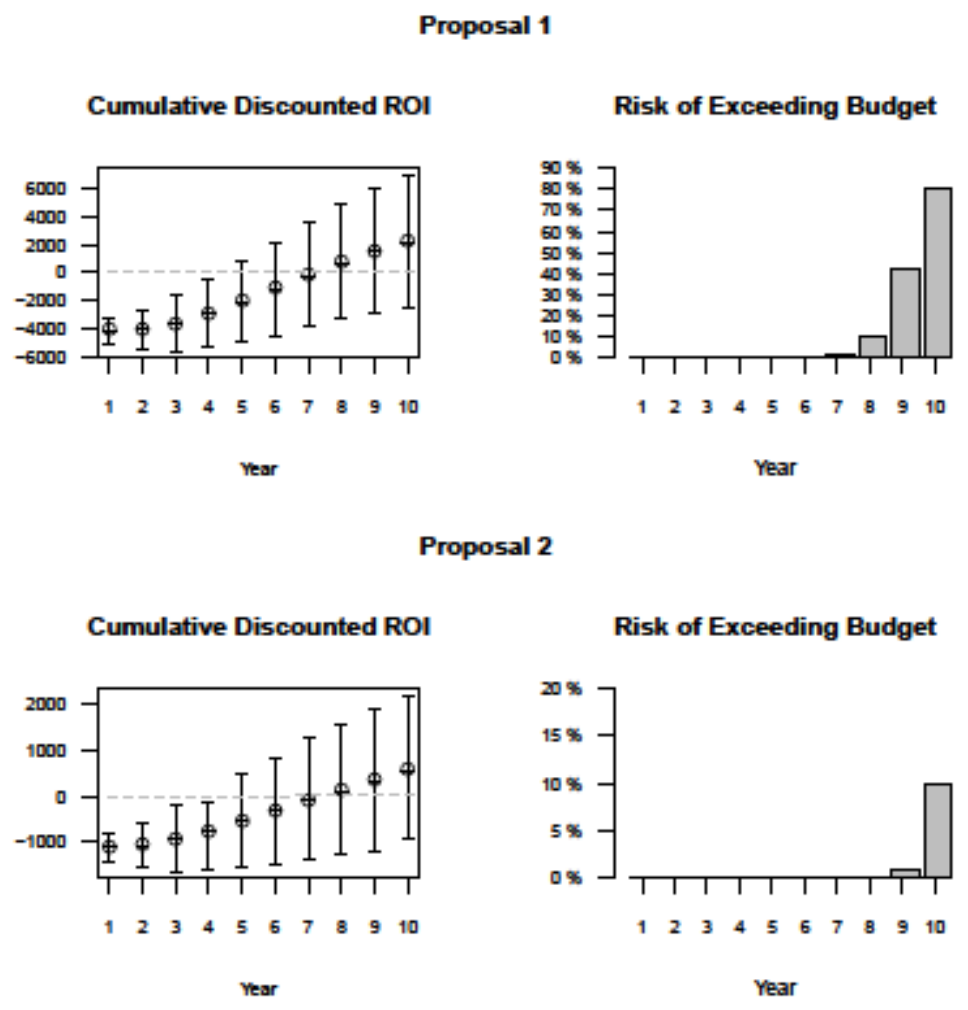

Figure 8 Cumulative DROI and REB for (a) Proposal 1 (b) Proposal 2

\subsection{Risk Scenarios}

The model offers a powerful way of analysing project risk scenarios as it is able to update the probability distributions of its variables when an observation is entered in any risk factor or variable in the model. In this section, we analyse the impact of two risk scenarios.

\subsubsection{Scenario 1: Incorrect Adoption Rate Estimate}

In the first scenario, the adoption rate estimate turns out to be incorrect as the true value of the rate of innovation and imitation is $10 \%$ and $15 \%$ lower than the expected values of the initial estimates respectively. For proposal 1, we entered 0.15 and 0.25 as observations to the rate of innovation $p$ and rate of imitation $q$ respectively. For proposal 2 we entered 0.20 and 0.35 to $p$ and $q$. Note that the expected values of $p$ and $q$ 's initial estimates were respectively 0.25 and 0.40 in proposal 1 , and 0.30 and 0.50 in proposal 2. 
Figure 9 shows the cumulative DROI for each proposal under this scenario. A lower adoption rate decreased both the project impact and the direct costs. As a result of the decreasing direct costs, the risk of exceeding budget is smaller compared to the initial analysis. The expected DROI of both proposals decreased significantly. The expected DROI of proposal 1 became negative as the impact significantly decreased; however the expected DROI of proposal 2 is still positive.

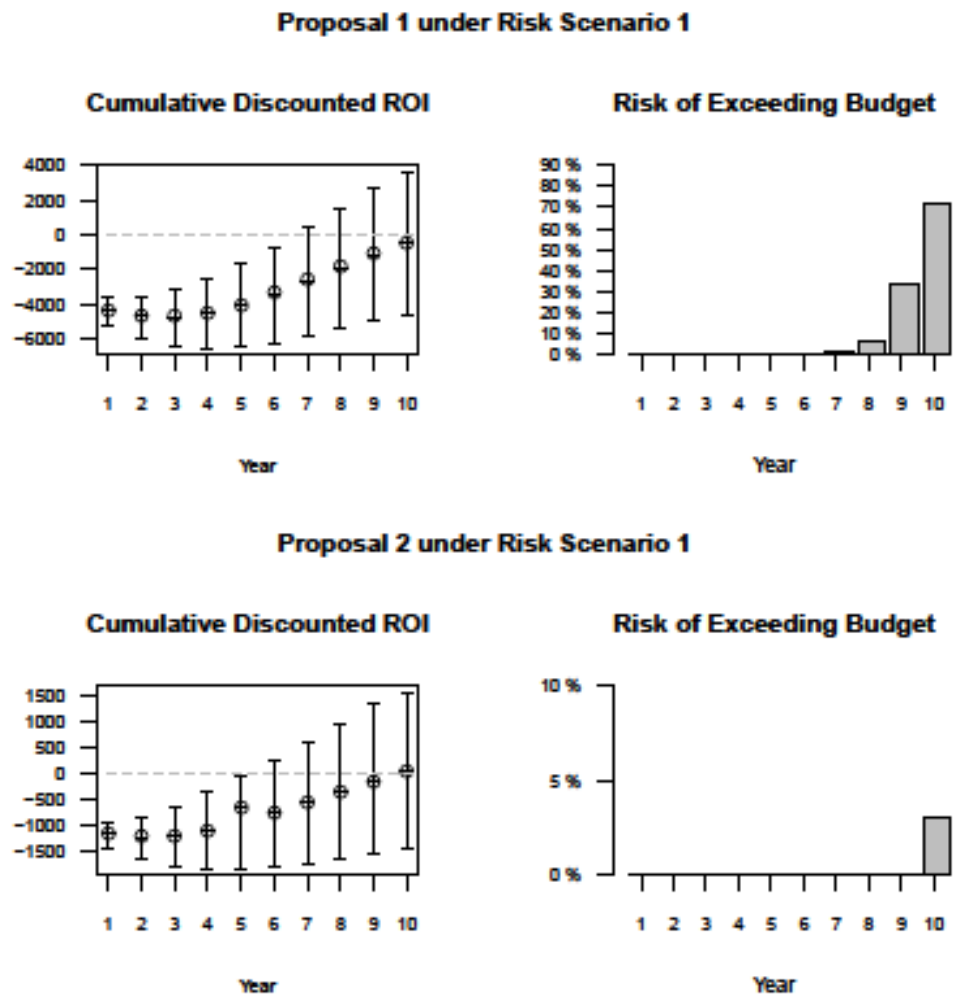

Figure 9 DROI and REB of (a) Proposal 1 (b) Proposal 2 under Risk Scenario 1

\subsubsection{Scenario 2: Multiple Risk Events}

In the second scenario, we entered observations for the following risk events and recalculated the probability distributions of each proposal:

- Extreme climate events observed in year 5 and 7

- Decreased lead institution efficacy and degree of involvement of enabling institution between years $5-10$.

- Decreased community involvement in years $8-10$. 
Proposal 1 under Risk Scenario 2

Cumulative Discounted ROI

Risk of Exceeding Budget

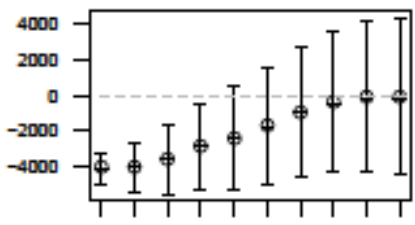

$\begin{array}{lllllllllll}1 & 2 & 3 & 4 & 5 & 6 & 7 & 8 & 9 & 10\end{array}$

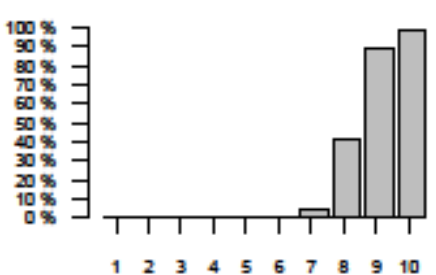

vear

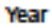

Proposal 2 under Risk Scenario 2

Cumulative Discounted ROI

Risk of Exceeding Budget

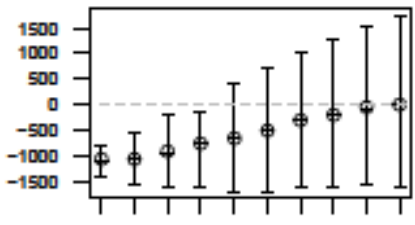

$\begin{array}{lllllllllll}1 & 2 & 3 & 4 & 5 & 6 & 7 & 8 & 9 & 10\end{array}$

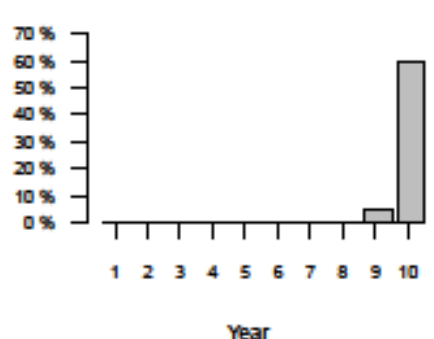

Figure 10 DROI and REB of (a) Proposal 1 (b) Proposal 2 under Risk Scenario 1

Figure 10 shows each proposal's cumulative DROI and risk of exceeding budget for this risk scenario.

The risk events increased the project costs and decreased the chance of having a positive DROI. In proposal 1, there is $99 \%$ risk of exceeding budget, and unlike the initial analysis, the project has a negative expected DROI at the end of the $10^{\text {th }}$ year. In proposal 2 , the expected DROI also significantly decreased but it still has a positive expected outcome in the $10^{\text {th }}$ year. In the following section, we analyse whether increasing budget could increase DROI of proposals in these scenarios.

\subsection{Budget Increase}

Exceeding the budget causes the impact of the projects to be lower than initially estimated. In this section we analyse the effect of investing additional funds to each proposal's budget in the first and second risk scenarios. 


\subsubsection{Additional Budget for Scenario 1}

In the first risk scenario (see Section 4.2.1), DROI decreases since the adoption rate, and thereby the impact, turns out to be lower than the initial estimate. Figure 11 shows the results of investing additional budget for proposal 1 and 2 in this case. We analysed the effects of investing an additional 4000 KUSD to the proposal 1 and 1000 KUSD to the proposal 2. The amount of additional budget was defined based on the $99^{\text {th }}$ percentile of the total cost in the $10^{\text {th }}$ year. Investing additional budget slightly increases the DROI in scenario 1 but its effects are minimal. In proposal 1, the DROI is still negative after the additional budget. DROI only increased by 10 KUSD in proposal 2. This is because the main cause of decreasing DROI was the decreasing impact, not increasing costs. Therefore, additional budget does not change the outcome significantly.

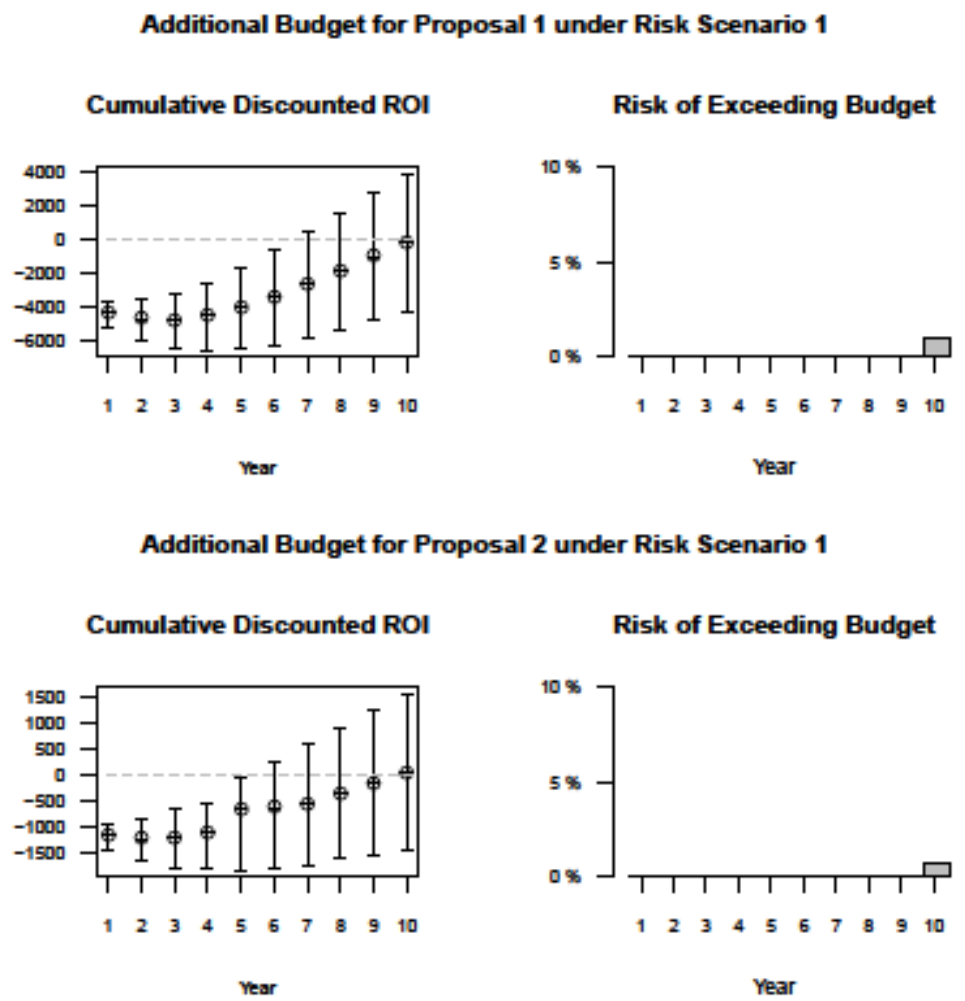

Figure 11 Additional Budget in Scenario 1 for (a) Proposal 1 and (b) Proposal 2 

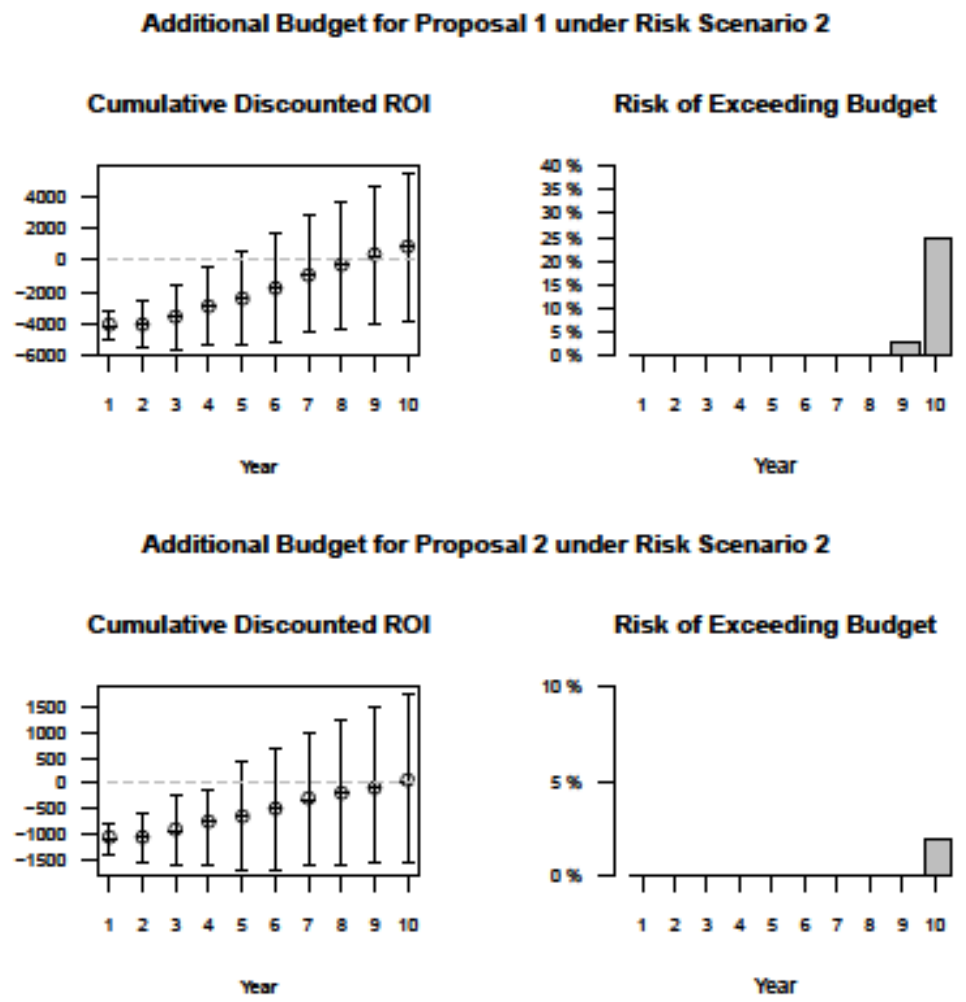

Figure 12 Additional Budget in Scenario 2 for (a) Proposal 1 and (b) Proposal 2

\subsubsection{Additional Budget for Scenario 2}

In the second risk scenario (see Section 4.2.2), we increased the budget by the same amount as in the first scenario. The risk events in scenario 2 increase the costs. The risk of exceeding the budget also increases due to increased costs. Exceeding the budget causes the impact of the project to be lower than initially estimated. When we invest additional funds to the projects' budget in scenario 2 the risk of running over budget decreases considerably. In proposal 1, the expected DROI of the project becomes positive starting from the $9^{\text {th }}$ year. In proposal 2, additional budget causes fivefold increase in the expected project DROI. Figure 12 shows the risk of exceeding budget and DROI when the budget is increased.

\subsection{Summary of the Results}

Our BN model successfully calculated the expected values and uncertainty of cost, impact and ROI of projects 1 and 2. Project 1 had a higher expected ROI than project 2 but its uncertainty and the risk of exceeding the budget were also higher. The uncertainty of all parameters and previous years were taken into account when predicting the costs and returns at a time stage. The uncertainty of ROI predictions 
increased in later years as long-term predictions are more uncertain than short-term predictions. The $\mathrm{BN}$ model enabled us to make a detailed what-if analysis of various risk factors happening at different stages. Project 2 was more robust to increased costs and decreased returns due to risk events and incorrect adoption rate predictions. Our model also enabled us to examine different budget policies as it calculated the effect of investing extra budget when risk events occur. Extra budget led to positive ROI for both projects but project 1 still had higher benefits. The main limitation of our framework was to assume a fixed time horizon. Although risk events and budget extension may have effects on the duration of a project, we used a 10-year horizon to analyse both projects.

\subsection{Use of Framework in Different Domains}

The BN template, with its underlying causal associations, should be applicable or readily adapted to most project situations. However, we expect that project managers will initially need to work with a trained decision analyst who is familiar with BNs to identify and include the individual variables contributing to the aggregated project impacts and risks, and construct their NPTs. Knowledge and experience is also required to construct probability distributions from data that is often form disparate sources and collected under different situations in which it is being applied (Constantinou et al., 2016, Yet et al., 2014a), and to elicit probability distributions from expert knowledge while avoiding the many types of biases that can occur (O'Hagan et al., 2006, Fenton and Neil, 2012, Tversky and Kahneman, 1974). However, this framework should make it easier for decision analysts to apply BNs to project management, in a similar way that the reusable BN idioms provided by Neil et al. (2000) help to model the structure of a risk assessment problem.

Since projects in different domains can have different cost, impact and risk factors, the parts of the BN framework relevant to these factors should be adapted to the domain where the framework is applied. This, however, does not require major changes to the overall causal associations of the framework. In the following section, we illustrate how our BN framework is applied to a different domain by using an 
example of a process development project. We use an extended version of the example described by Hess (1993).

\subsubsection{Use of Framework for a Process Development Project}

A chemical company has developed an agent called antraquinone for reducing pulp mill water pollution. This agent can potentially be a commercial success if the company develops a new synthesising process for it. The main risks that may delay or fail the project are the following:

- Technical risk: the process may not be developed,

- Market risk: estimated marked demand may not be accurate,

- Commercial risk: the venture may not be commercially successful,

- Lack of board support: board may cease their support for the project.

The overall impact of the project is expected to be 7 million dollars per year when the project is fully adopted by potential customers. The main costs elements of this project are process development, research, market development, commercial development and yearly upkeep expenses.

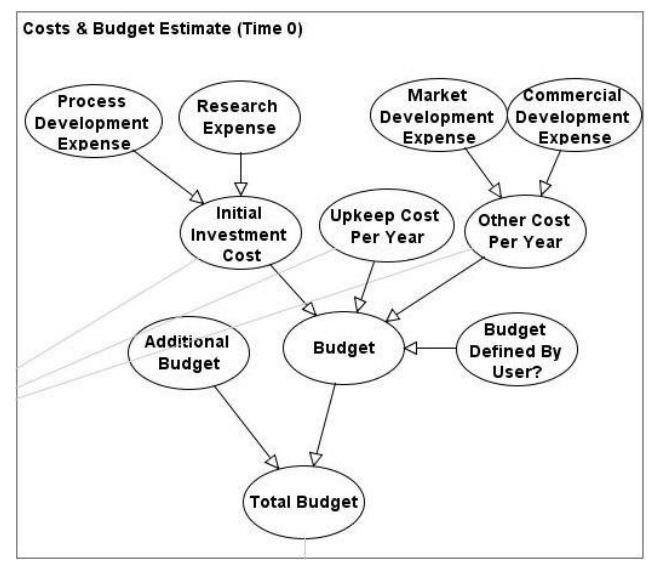

Figure 13 Modified Cost \& Budget Estimate Fragment for Process Development Project Example 


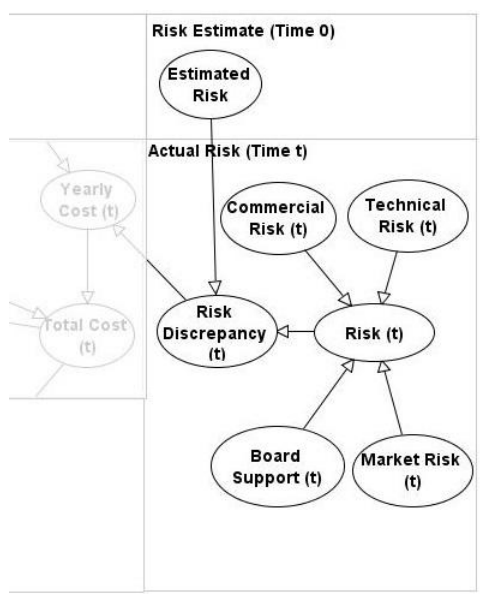

Figure 14 Modified Actual Risk Fragment for Process Development Project Example

In order to apply our BN framework to this project, we first modified the 'Project Impact Estimate', 'Cost \& Budget Estimate' and 'Actual Risk at time t' components according to the specifics of the project. Only the 'Target Project Impact' variable was modelled in the 'Project Impact Estimate' component as different impact categories were not defined in this example. Next, the main costs elements of this example had to be classified as either 'Initial Investment Cost' or 'Upkeep Cost' or 'Other Cost' to model them in the 'Cost \& Budget Estimate' component. We classified process development and research expenses as initial investment costs, and market development and commercial expenses as other costs. We added these variables as parents of the relevant cost classifications (see Figure 13). We added the individual risk factors as parents of the 'Risk (t)' variables in the 'Actual Risk at time t' components in all time stages (see Figure 14). We also removed the 'Country' variable from the 'Risk Estimate' fragment as this variable is not relevant to this domain. Note that, FDR, rather than EDR, was used as the discount factor in this example, therefore the value of MEDR was set to 0 . After modifying the structure for this example, we entered the input parameters shown in Table 2, and calculated DROI and REB over a 5 year period (see Figure 15). The unit of all monetary values in Table 2 is in millions US dollars. The expected DROI of the process development project was 9.4 million US dollars, and REB was $12.5 \%$ at the end of fifth year. 
Table 2 Input Values and Distributions for Process Development Project Example

\begin{tabular}{ll}
\hline \multicolumn{1}{c}{ Variable } & \multicolumn{1}{c}{ Input Values / Distributions } \\
\hline Initial Budget & 14 \\
Initial Investment Cost & Research Expense + Process Development Expense \\
Upkeep Cost / Year & TNormal $(0.4,0.05,0,2)$ \\
Other Cost / Year & Commercial Development Expense + Market Development Expense \\
Research Expense & LogNormal $(-0.2,0.45)$ \\
Process Development Expense & TNormal $(3,0.6,0,20)$ \\
Market Development Expense / Year & TNormal $(0.2,0.001,0,5)$ \\
Commercial Development Expense / Year & TNormal $(1,0.1,0,10)$ \\
Target Project Impact / Year & 7 \\
Risk estimation & Low \\
Rate of innovation $(\boldsymbol{p})$ & $\operatorname{Beta}(3,7)$ \\
Rate of imitation $(\boldsymbol{q})$ & $\operatorname{Beta}(40,60)$ \\
ADR & $\operatorname{TNormal}(0.03,0.001,0,1)$ \\
MEDR & 0 \\
\hline
\end{tabular}

We did not need to change the overall causal relations in our framework to use it for the process development project example. We only modified 'Project Impact Estimate', 'Cost \& Budget Estimate' and 'Actual Risk at time t' fragments according to the specific cost, impact and risk factors of this project.

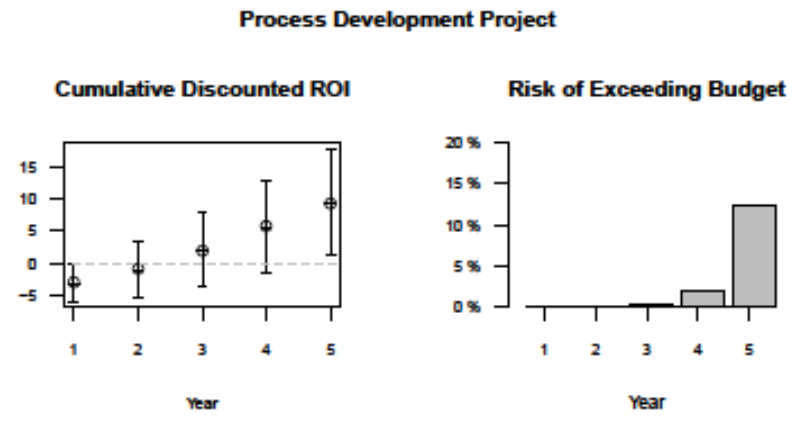

Figure 15 DROI and REB of Process Development Project over 5 years

\section{Conclusion and Future Work}

This paper presented a $\mathrm{BN}$ modelling framework that calculates costs, benefits and ROI of projects by taking budget, individual risk factors, adoption and discount rate into account. The framework offers a powerful method for analysing risk scenarios and their effects on project success and does so using a DBN that uses standardised components and reusable template models. The underlying BN models use the causal and associational relations between the parameters, and take uncertainty into account while making calculations. The model uses the entire probability distribution of both discrete and continuous variables when calculating the posteriors. The method is illustrated using a case study of an agricultural 
development project. The case study demonstrated the relative ease with which the generic framework could be tailored to a specific application domain, and also the powerful range of quantitative risk insights under different scenarios and assumptions.

Our method can potentially help the decision makers during project selection, planning and control stages of project management. In the selection stage, the method can be used to make cost-benefit analysis of project alternatives. In the planning stage, it can be used to estimate the costs, impact and budget. The method can also be used to analyse risk at that stage by calculating the potential effects and the degree of uncertainty of different risk factors. In the control stage, users can update the model and revise the plan as the project progresses. They can enter observed costs, impact, and risk factors as the project continues, and revise the predictions about the project outcomes. The iterative updating of projections with evidence also provides a learning and impact evaluation tool.

Any process to improve project management implies an additional investment of time and resources and there is considerable uncertainty over the returns on investment from improvement processes such as project maturity management. Mishra et al. (2016) found for US federal technology projects that investment in higher project maturity levels was important for attenuating the negative effects of project risks on performance at high risk levels, but at low risk levels higher maturity had negative impacts on schedule and cost metrics. In the context of development projects, the largest uncertainties are often not so much in whether time and cost over-runs will occur but rather in whether the projects will achieve the intended outcomes (e.g., due to slow adoption rates) and in the area of project implementation risks (e.g., Luedeling et al. 2015). In such cases, there may be considerable benefit generated from quantifying the uncertainty in the project impact pathway, in terms of insights into how to improve project design to maximize the probability of achieving favourable outcomes and minimize implementation risks, as well from identifying areas for adjustment during project control stages and for impact evaluation (Shepherd et al., 2015). In addition, the BN modelling process encourages a holistic approach whereby all factors deemed important to the project's success are quantified, rather than ignoring them because of large 
uncertainties (e.g., offsite environmental impacts). The potential of the framework to considerably improve on the common use of risk registers (Fenton and Neil, 2012) should also increase incentives for use of the BN framework. If these benefits can be demonstrated more widely to project managers and organizations, then this could provide an incentive for investment in BNs as a component of maturity management.

The case study showed the results of the method when multiple risk factors and budget extension are analysed for proposals of two alternative agricultural development projects. The application of our modelling framework, however, is not limited to agricultural projects. The general structure shown in Figure 1 and Figure 2 can be instantiated and specialised for a wide variety of domains such as construction and software projects. Moreover, the model can be extended by adding more detailed components or modifying the existing components. Ways to instantiate the framework for other domains were illustrated by using an example of a process development project.

Because the existing techniques about cost and budget estimation do not incorporate the extent of information that our framework allows, it is not useful to compare the results/accuracy of our models to those. Moreover because of the very uncertainty of project planning, and the inevitable lack of sufficiently large and relevant datasets, traditional 'validation' of the framework and models is unrealistic.

As further research, we plan to develop an efficient value of information technique (VoI) for BNs with continuous variables. Chapman and Ward (2004) argue that “... best practice in project risk management is concerned with managing uncertainty that matters in an effective and efficient manner." VoI is a useful technique to identify uncertainty that matters in an efficient way as it identifies the parts of a model where additional investment is most useful. Therefore, VoI enables the user to focus where uncertainty reduction is most valuable (Coyle and Oakley, 2008). However, making a VoI analysis for individual continuous variables is a challenging task often requiring complex approximations (Madan et al., 2014). An efficient and convenient way of analysing the VoI of individual variables would be a useful complementary technique for our framework. We also plan to implement the uncertainty of time aspect to our modelling 
framework. Our framework currently assumes a fixed time period for a project but time schedules of projects are highly uncertain. We plan to extend our framework with the time uncertainty aspect of Khodakarami et al. (2007). Moreover, our BN framework could be extended to model costs, benefits and risks of different stakeholders. Including time uncertainty and views of different stakeholders would be a step towards having a unified modelling framework for all subjects of project risk analysis.

Finally, we plan to make our framework more user-friendly for project managers who are not experts in BN technology. Our model must be modified and instantiated in order to apply it to projects from other domains. Currently, this requires knowledge of $\mathrm{BN}$ models and software. We aim to develop general building blocks for project management models using a similar approach to the BN idioms (Neil et al., 2000). This would enable users to easily develop and modify their own models by simply selecting the building blocks that are suitable to their domain.

\section{Acknowledgements}

Part of this work was performed under the auspices of ICRAF Contract No SD4/2012/214 issued to Agena and part under EU project ERC-2013-AdG339182-BAYES_KNOWLEDGE. We acknowledge support from the Water, Land and Ecosystems (WLE) program of the Consultative Group on International Agricultural Research (CGIAR).

\section{References}

AGENARISK. 2015. AgenaRisk: Bayesian network and simulation software for risk analysis and decision support [Online]. Available: http://www.agenarisk.com [Accessed 02.01.2015 2015].

BACKLUND, F., CHRONÉER, D. \& SUNDQVIST, E. 2014. Project Management Maturity Models-A Critical Review: A Case Study within Swedish Engineering and Construction Organizations. Procedia-Social and Behavioral Sciences, 119, 837-846.

BASS, F. M. 1969. A New Product Growth for Model Consumer Durables. Management science, 15, 215 - 227.

CHAPMAN, C. \& WARD, S. 2004. Why risk efficiency is a key aspect of best practice projects. International Journal of Project Management, 22, 619-632 \%@ 0263-7863.

CHENG, J., BELL, D. \& LIU, W. 1998. Learning Bayesian networks from data: An efficient approach based on information theory. On World Wide Web at http://www. cs. ualberta. ca/ jcheng/bnpc. htm.

CONSTANTINOU, A. C., FENTON, N., MARSH, W. \& RADLINSKI, L. 2016. From complex questionnaire and interviewing data to intelligent Bayesian Network models for medical decision support. Artificial Intelligence in Medicine, 67, 75-93.

CONSTANTINOU, A. C., FENTON, N. E. \& NEIL, M. 2012. pi-football: A Bayesian network model for forecasting Association Football match outcomes. Knowledge-Based Systems, 36: 322-339. 
CONSTANTINOU, A. C., FENTON, N. E. \& NEIL, M. 2013. Profiting from an inefficient Association Football gambling market: Prediction, Risk and Uncertainty using Bayesian networks. Knowledge-Based Systems, $50,60-86$.

COYLE, D. \& OAKLEY, J. 2008. Estimating the expected value of partial perfect information: a review of methods. The European Journal of Health Economics, 9, 251-259.

DALY, R., SHEN, Q. \& AITKEN, S. 2011. Learning Bayesian networks: approaches and issues. The knowledge engineering review, 26, 99-157.

DE MELO, A. C. \& SANCHEZ, A. J. 2008. Software maintenance project delays prediction using Bayesian Networks. Expert Systems with Applications, 34, 908-919.

FAN, C.-F. \& YU, Y.-C. 2004. BBN-based software project risk management. Journal of Systems and Software, 73, 193-203.

FENTON, N., BERGER, D., LAGNADO, D., NEIL, M. \& HSU, A. 2014. When 'neutral' evidence still has probative value (with implications from the Barry George Case). Science \& Justice, 54, 274-287.

FENTON, N., MARSH, W., NEIL, M., CATES, P., FOREY, S. \& TAILOR, M. Making resource decisions for software projects. 2004. IEEE, 397-406\%@0769521630.

FENTON, N. \& NEIL, M. 2012. Risk assessment and decision analysis with Bayesian networks, CRC Press.

FENTON, N., NEIL, M. \& LAGNADO, D. A. 2013. A general structure for legal arguments about evidence using Bayesian networks. Cognitive science, 37, 61-102.

FENTON, N. E. \& NEIL, M. 2014. Decision Support Software for Probabilistic Risk Assessment Using Bayesian Networks. IEEE software, 31, 21-26.

FENTON, N. E., NEIL, M. \& CABALLERO, J. G. 2007. Using ranked nodes to model qualitative judgments in Bayesian networks. Knowledge and Data Engineering, IEEE Transactions on, 19, 1420-1432.

FINEMAN, M., FENTON, N. \& RADLINSKI, L. Modelling project trade-off using Bayesian networks. 2009. IEEE, 1-4\%@1424445078.

GREEN, S. D. 2001. Towards an integrated script for risk and value management. Project management, 7, 52-58.

HECKERMAN, D. 1997. Bayesian networks for data mining. Data mining and knowledge discovery, 1, 79-119.

HESS, S. W. 1993. Swinging on the branch of a tree: project selection applications. Interfaces, 23, 5-12.

HU, Y., ZHANG, X., NGAI, E., CAI, R. \& LIU, M. 2013. Software project risk analysis using Bayesian networks with causality constraints. Decision Support Systems, 56, 439-449.

KERZNER, H. R. 2001. Strategic planning for project management using a project management maturity model, New York, John Wiley \& Sons.

KHODAKARAMI, V. \& ABDI, A. 2014. Project cost risk analysis: A Bayesian networks approach for modeling dependencies between cost items. International Journal of Project Management, 32, 1233-1245.

KHODAKARAMI, V., FENTON, N. \& NEIL, M. 2007. Project Scheduling: Improved approach to incorporate uncertainty using Bayesian Networks. Project Management Journal, 38, 39.

KOLLER, D. \& FRIEDMAN, N. 2009. Probabilistic graphical models: principles and techniques, MIT press.

LEE, E., PARK, Y. \& SHIN, J. G. 2009. Large engineering project risk management using a Bayesian belief network. Expert Systems with Applications, 36, 5880-5887.

LUEDELING, E., OORD, A. L., KITEME, B., OGALLEH, S., MALESU, M., SHEPHERD, K. D. \& DE LEEUW, J. 2015. Fresh groundwater for Wajir - ex-ante assessment of uncertain benefits for multiple stakeholders in a water supply project in Northern Kenya. Frontiers in Environmental Science, 3, 16.

LUU, V. T., KIM, S.-Y., VAN TUAN, N. \& OGUNLANA, S. O. 2009. Quantifying schedule risk in construction projects using Bayesian belief networks. International Journal of Project Management, 27, 39-50.

MADAN, J., ADES, A. E., PRICE, M., MAITLAND, K., JEMUTAI, J., REVILL, P. \& WELTON, N. J. 2014. Strategies for Efficient Computation of the Expected Value of Partial Perfect Information. Medical Decision Making.

MISHRA, A., DAS, S. R. \& MURRAY, J. J. 2016. Risk, Process Maturity, and Project Performance: An Empirical Analysis of US Federal Government Technology Projects. Production and Operations Management, 25, 210-232.

NEIL, M., FENTON, N. \& NIELSON, L. 2000. Building large-scale Bayesian networks. The Knowledge Engineering Review, 15, 257-284.

NEIL, M., HAGER, D. \& ANDERSEN, L. B. 2009. Modeling operational risk in financial institutions using hybrid dynamic Bayesian networks. Journal of Operational Risk, 4, 3-33. 
NEIL, M., TAILOR, M. \& MARQUEZ, D. 2007. Inference in hybrid Bayesian networks using dynamic discretization. Statistics and Computing, 17, 219-233.

O'HAGAN, A., BUCK, C. E., DANESHKHAH, A., EISER, J. R., GARTHWAITE, P. H., JENKINSON, D. J., OAKLEY, J. E. \& RAKOW, T. 2006. Uncertain judgements: eliciting experts' probabilities, Chichester, John Wiley \& Sons.

PEARL, J. 1988. Probabilistic reasoning in intelligent systems: networks of plausible inference, Morgan Kaufmann.

PEARL, J. 2000. Causality: models, reasoning and inference, Cambridge Univ Press.

PERKUSICH, M., SOARES, G., ALMEIDA, H. \& PERKUSICH, A. 2015. A procedure to detect problems of processes in software development projects using Bayesian networks. Expert Systems with Applications, 42, 437-450.

PMI 2013. A guide to the project management body of knowledge (PMBOK guide), Newtown Square, PA, Project Management Institute.

RENOOIJ, S. 2001. Probability elicitation for belief networks: issues to consider. The Knowledge Engineering Review, 16, 255-269.

SAVAGE, S. L. 2012. The flaw of averages. Why we underestimate risk in the face of uncertainty, Hoboken, NJ, John Wiley \& Sons.

SHEPHERD, K., HUBBARD, D., FENTON, N., CLAXTON, K., LUEDELING, E. \& DE LEEUW, J. 2015. Policy: Development goals should enable decision-making. Nature, 523, 152.

SPALEK, S. 2014. Does investment in project management pay off? Industrial Management \& Data Systems, 114, $832-856$.

TVERSKY, A. \& KAHNEMAN, D. 1974. Judgment under uncertainty: Heuristics and biases. science, 185, 11241131.

WARD, S. \& CHAPMAN, C. 2003. Transforming project risk management into project uncertainty management. International Journal of Project Management, 21, 97-105 \%@ 0263-7863.

WARD, S. C. \& CHAPMAN, C. B. 1995. Risk-management perspective on the project lifecycle. International Journal of Project Management, 13, 145-149\%@ 0263-7863.

WORLDBANK 2010. Cost-benefit analysis in World Bank projects. Washington DC: The World Bank.

WORLDBANK 2011. Impact evaluations in agriculture an assessment of the evidence. Washington DC: The World Bank.

YET, B., BASTANI, K., RAHARJO, H., LIFVERGREN, S., MARSH, W. \& BERGMAN, B. 2013. Decision support system for Warfarin therapy management using Bayesian networks. Decision Support Systems, 55, 488-498.

YET, B., PERKINS, Z., FENTON, N., TAI, N. \& MARSH, W. 2014a. Not just data: a method for improving prediction with knowledge. J Biomed Inform, 48, 28-37.

YET, B., PERKINS, Z. B., RASMUSSEN, T. E., TAI, N. R. M. \& MARSH, D. W. R. 2014b. Combining data and meta-analysis to build Bayesian networks for clinical decision support. Journal of Biomedical Informatics. 
